disturbances similar to that of autistic spectrum disorder. (Roulet Perez et al. Dev Med Child Neurol 1993;35:661-674).

\title{
AUTISTIC SPECTRUM DISORDER AND CHILDHOOD ONSET EPILEPSY
}

The prevalence of autistic spectrum disorder (ASD) among 519 patients with epilepsy, and the clinical characteristics of patients with both pathologies were analyzed retrospectively at Saga University, Japan. Of 79 (15.2\%) patients with epilepsy and ASD, 62 patients had idiopathic ASD and 17 had secondary ASD. Male preponderance was 47 to 15 females; median age 11 years (range 2 to 43 years). Age at onset of seizures was most frequently 4 years; $85 \%$ occurred before 10 years of age. ASD was detected after onset of epilepsy in 29 cases $(46.8 \%)$, and the diagnosis was delayed for $>5$ years in 8 , mostly high-functioning ASD cases. Complex partial seizure was the most frequent pattern, occurring in $68 \%$. Paroxysmal activities in the EEG were frontal in half the cases. Response to AEDs was complete for more than 2 years in $67.3 \%$ patients. Autistic symptoms improved after epilepsy treatment in 5 cases $(8 \%)$. (Matsuo M, Maeda T, Sasaki K, Ishii K, Hamasaki Y. Frequent association of autistic spectrum disorder in patients with childhood onset epilepsy. Brain Dev Oct 2010;32:759-763). (Respond: Muneaki Matsuo MD, Dept Pediatrics, Saga University, Japan. E-mail: matsuo@cc.sagau.ac.jp).

COMMENT. Epilepsy associated with ASD is usually characterized by complex partial seizures with frontal paroxysms with onset from 1 to 9 years of age. The prevalence of epilepsy with ASD in this study was $15 \%$, lower than some previous reports of $20 \%$ to $46 \%$ in different populations.

An International Symposium on Epilepsy in ASDs and related conditions, edited by Dr Yukio Fukuyama and colleagues, was the topic at the $12^{\text {th }}$ Annual Meeting of the Infantile Seizure Society held in Kurume, Japan, May 9-10, 2009. Several review and original articles are published in the October issue of Brain Dev 2010;32:695-780).

Dr Amy Brooks-Kayal of University of Colorado Denver School of Medicine reviews the common genetic, molecular and cellular developmental mechanisms of ASD and epilepsy. (Brain Dev Oct 2010;32:731-738). Genetic disorders sharing epilepsy and autism include tuberous sclerosis, Rett syndrome, and fragile X. Various mutations of several genes involved in neurodevelopment are found in both disorders. Early life seizures can result in cellular and molecular changes in the hippocampus that contribute to learning and behavior disorders as seen in ASD. The excitatory-inhibitory imbalance resulting from these factors may represent new therapeutic targets for treatment.

\section{NEUROPSYCHOLOGICAL SYMPTOMS AND PREDICTION OF SEIZURE RECURRENCE}

Researchers at the Departments of Medicine, Psychiatry, Neurology and Paediatrics, University of Melbourne, Australia investigated the predictive value of neuropsychiatric symptomatology in control of seizures in patients with epilepsy newly 
treated with AEDs. The majority of the patients had localization related epilepsy. A neuropsychological assessment scale (ABNAS) was completed by 170 newly treated patients with epilepsy followed prospectively for 12 months. The ABNAS is a validated brief scale of cognitive and behavioral function that correlates with scales for memory, anxiety, and depression levels. A higher score, reflecting greater neuropsychiatric symptomatology and potentially more widespread brain dysfunction, is associated with AED unresponsiveness. Of 138 with a drug response phenotype at 12 months, 45 nonresponsive patients (at least 1 seizure) had a higher pretreatment ABNAS score than 93 whose seizures were controlled $(\mathrm{p}=0.007)$. Patients with a low pretreatment ABNAS score $(<15)$ were more likely to be pharmacoresponsive at 12 months than those with a high ABNAS score $(>15)(72 / 100$ [72\%] vs 21/38 [55\%], $\mathrm{p}=0.049)$. A higher risk of seizure recurrence was also associated with a lesion on MRI $(p=0.003)$. For a AED pharmacoresponse, a multivariate model (ABNAS, MRI, and genotype profile classifier) had diagnostic values of $91 \%$ sensitivity, $64 \%$ specificity, $84 \%$ positive predictive, and $78 \%$ negative predictive values. The predictive value of the ABNAS score was also validated in a second prospective cohort of 74 newly treated patients with epilepsy ( $\mathrm{p}=0.005$ ). (Petrovski S, Szoeke CEI, Jones NC, et al. Neuropsychiatric symptomatology predicts seizure recurrence in newly treated patients. Neurology Sept 14, 2010;75:10151021). (Respond and reprints: Dr Terence J O'Brien, Department of Medicine, Royal Melbourne Hospital, University of Melbourne, Royal Parade, Parkville, 3050, Victoria, Australia. E-mail: obrientj@unimelb.edu.au).

COMMENT. A poor response to AEDs in newly diagnosed patients with epilepsy may be predicted by a high score on a pretreatment neuropsychological assessment scale, and MRI evidence of structural brain lesion and genomic factors also contribute to a multifactorial AED response phenotype. At least $30 \%$ to $40 \%$ of patients newly treated for seizures have further seizures despite appropriate AED therapy (Kwan P, Brodie MJ. N Engl J Med 2000;342:314-319; cited by authors). The above study, mainly in adults, mean age 39 years, demonstrates the importance of neuropsychological and genomic data in prediction of response of newly diagnosed epilepsy to AEDs. Neuropsychological testing and genomic typing are also assuming more prominent roles in the management of pediatric epilepsy. Of a total of 212 patients in the above study, 32 (15\%) were children and adolescents, mean age 16, range 10-18 years. (per Dr Petrovski).

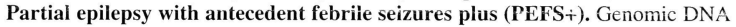
from 4 patients with PEFS+ was screened for mutations in SCN1A, SCN2A, SCN1B, and GABRG2. Two heterozygous de novo mutations of SCN1A were detected that caused loss of function of Nav1.1, and were associated with seizure aggravation by AEDs. PEFS+ is similar to SMEI and GEFS+ clinically with sporadic onset and possible AED-induced seizure aggravation. Genotyping is helpful in the management of these epilepsies. (Liao W-P et al. Epilepsia Sept 2010;51(9):1669-1678).

\section{PRADER-WILLI SYNDROME AND ATONIC SEIZURES}

A 2.5-year-old boy with Prader-Willi syndrome and a history of neonatal superior sagittal sinus thrombosis developed a febrile seizure and new onset atonic drop seizures originating in the parasagittal region, as reported from Children's Hospital, Boston. A 